\title{
A rare case of primary diffuse large B-cell lymphoma of the colon
}

\author{
Muhammad Tahir ${ }^{1}$, Kashif Samad ${ }^{2}$, Thomas Koenig ${ }^{3}$, Prakash Viswanathan ${ }^{3}$ \\ ${ }^{1}$ Department of Internal Medicine, Sisters of Charity Hospital, Buffalo, NY 14214, USA; ${ }^{2}$ Internal Medicine, Sisters of Charity, University at Buffalo, \\ Buffalo, NY 14214, USA; ${ }^{3}$ Department of Gastroenterology, North Memorial Hospital, MN, USA \\ Correspondence to: Muhammad Tahir, MD. Department of Internal Medicine, Sisters of Charity Hospital, 2157 Main St, Buffalo, NY 14214, USA. \\ Email: muhammadtahir090@gmail.com.
}

\begin{abstract}
The gastrointestinal tract is the most common site in the United States for extra-nodal lymphoma involvement. Primary colorectal involvement is very rare, only accounting for $0.3 \%$ of the large intestine cancers. There has been a small increase in the incidence in these high-risk patient populations which include IBD, Celiac disease, H. pylori and other autoimmune diseases. We report a case of 47 years old male with no risk factors, who presented to the hospital with non-specific signs and symptoms. Imaging revealed cecal mass with distended bowel and colonoscopy revealed large mass obstructing the whole lumen of the cecum. The histopathology showed diffuse large B-cell lymphoma and patient underwent hemicolectomy. Chemotherapy was started and the patient improved.
\end{abstract}

Keywords: Lymphoma; colon; primary colonic tumor

Received: 05 May 2018; Accepted: 14 May 2018; Published: 01 June 2018.

doi: 10.21037 /acr.2018.05.04

View this article at: http://dx.doi.org/10.21037/acr.2018.05.04

\section{Introduction}

The gastrointestinal tract is the most common site in the United States for extra-nodal lymphoma involvement. Primary colorectal involvement is very rare, only accounting for $0.3 \%$ of the large intestine cancers. Several etiologies have been identified which been shown to predispose lymphoma formation. There has been a small increase in the incidence in these high-risk patient populations which include IBD, Celiac disease, H. pylori and other autoimmune disease. Due to the rarity of primary intestinal disease, it is important to understand the presentation, course, progression and management of this disease.

Here we report a case of middle-aged low risk male patient who was diagnosed with colonic diffuse large B-cell lymphoma (DLBCL).

\section{Case presentation}

The 47 years old male with no significant past medical history presented to Emergency room with the complaint of worsening RLQ abdominal pain for 1 month. Patient reported associated decreased appetite and weight loss but denied any hematochezia, melena, hematemesis, nausea, vomiting or fever. Physical examination revealed nondistending abdomen with palpable mass in right lower quadrant which was mildly tender to palpation but no guarding or rebound was appreciated. No palpable lymph nodes noted. CT Imaging revealed cecal mass-like material of mixed density $9.8 \times 12.4 \times 9.5 \mathrm{~cm}^{3}$ with distended small bowel. Lab workup revealed normal cell count, chemistry, negative PPD, normal CEA and CA 19-9. Colonoscopy was performed as shown in the Figure 1. Patient was found to have a large mass encompassing the whole lumen of cecum making it difficult to identify closely located landmarks. It was felt firm in texture during biopsy. The pathology report showed diffuse large B-cell lymphoma, germinal center B-cell subtype as seen in Figure 2. Patient underwent right hemicolectomy. Subsequently, patient was started on chemotherapy and is doing well till date.

\section{Discussion}

Primary colorectal lymphoma is very rare and accounts for $3 \%$ of the gastrointestinal lymphomas and $0.3 \%$ of the 


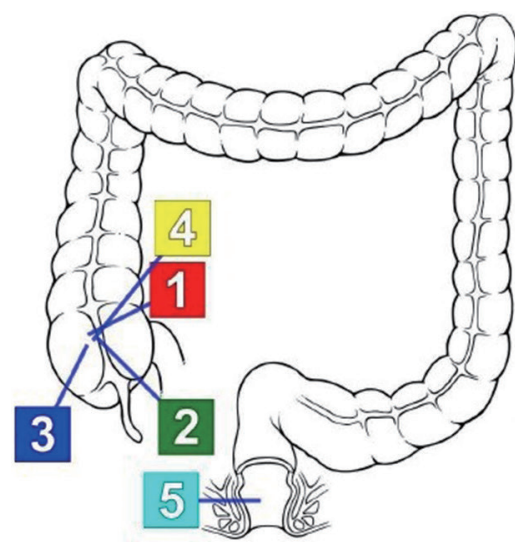

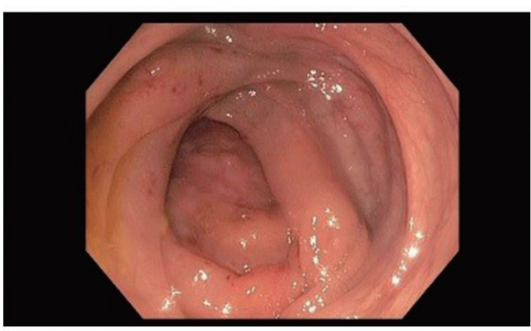

1 Cecum

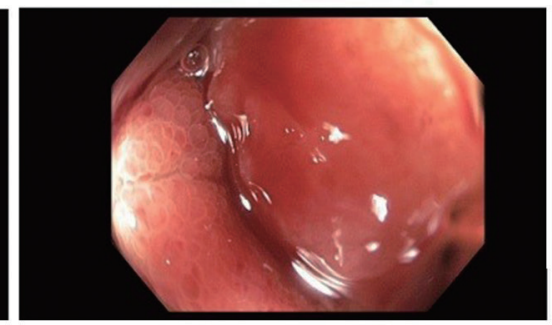

4 Cecum: mass

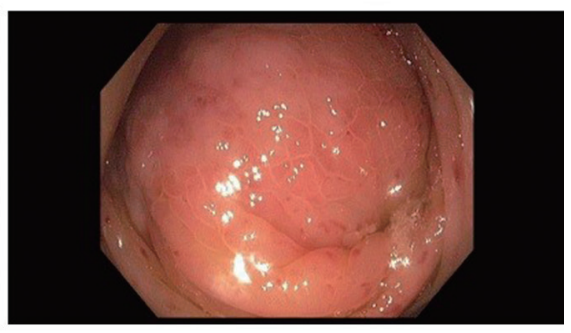

Cecum: mass

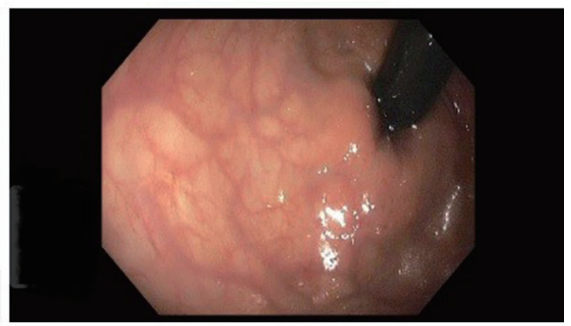

(5) Rectum

3 Cecum: mass

Figure 1 Colonoscopy showing cecal mass with moderate to severe encompassment of the lumen.
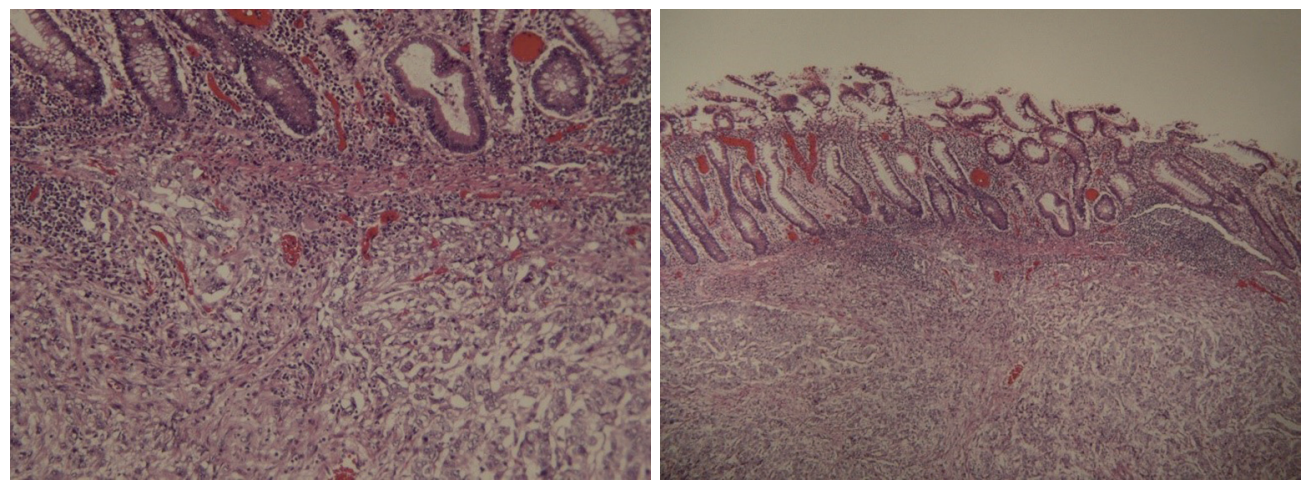

Figure 2 Histopathology showing diffuse large B-cell lymphoma, with germinal center, involving colonic wall (H/E stain, magnifications unknown).

cancers involving the large intestine (1). The literature has reported a male predominance usually in $6^{\text {th }}$ or $7^{\text {th }}$ decade. The most common histopathology type seen in colorectal involvement is DLBCL and mantle cell lymphoma.

The pathophysiology is still unknown, however there have been associated risk factors as discussed earlier. Despite the sever luminal narrowing, as seen in our patients, it rarely causes obstruction because it does not show a desmoplastic reaction and infiltration of the malignant cell weakens the muscularis propria of the colonic wall. There may be a resemblance in phenotypic appearance and correct diagnosis is often challenging (2). The presentation to the healthcare setting is non-specific and patients do present with occasional abdominal pain with or without bowel changes, and weight loss. Palpable abdominal mass as in this case can present in half of the patients. Bowel obstruction and perforation is a rare presentation as compared to colorectal adenocarcinoma.

The mainstay of the diagnosis is colonoscopy with biopsy. Different morphologies can be seen during endoscopy 
including diffuse mucosal nodularity, colitis like changes, ulceration or a mass with or without ulceration (3). Other modalities including CT imaging and barium enemas can also be helpful to localize the disease process and different pattern like focal nodularity, masses, fold or circumferential thickening (4). In our case CT imaging revealed a mass which was biopsied during the endoscopic intervention. There have been reports in literature where biopsy have been non-diagnostic and diagnosis was made after intestinal resection. The criteria for diagnosis of primary colonic lymphoma includes the disease being limited locally, with or without local lymph node involvement, but there should be absence of generalized or other systemic manifestations of the disease such as lymphadenopathy of the retroperitoneal or mediastinum. There also should be no signs of bone marrow or abdominal organ involvement (5).

There are many prognostic systems being used for DLBCL with "International Prognostic Index (IPI)" as the most common one. New techniques like gene expression profiling has been in use as well (6). Accurate staging is vital to stratify the management plan. Locoregional lymphadenopathy is also present in few cases at the time of diagnosis. The mainstay of treatment is surgical resection along with postoperative chemotherapy. Improvement in disease free period and survival rates has been seen due to current aggressive management. However, the disease ultimately leads to death due to recurrence in majority of the patients.

\section{Acknowledgements}

None.

doi: 10.21037/acr.2018.05.04

Cite this article as: Tahir M, Samad K, Koenig T, Viswanathan P. A rare case of primary diffuse large B-cell lymphoma of the colon. AME Case Rep 2018;2:28.

\section{Footnote}

Conflicts of Interest: The authors have no conflicts of interest to declare.

Informed Consent: Verbal consent was obtained. No identifier is used in the report.

\section{References}

1. Papaxoinis G, Papageorgiou S, Rontogianni D, et al. Primary gastrointestinal non-Hodgkin's lymphoma: a clinicopathologic study of 128 cases in Greece. A Hellenic Cooperative Oncology Group study (HeCOG). Leuk Lymphoma 2006;47:2140-6.

2. Risio D, Percario R, Legnini M, et al. Diffuse large B-cell lymphoma of the colon with synchronous liver metastasis: a rare case report mimicking metastatic colorectal adenocarcinoma. BMC Surg 2014;14:75.

3. Myung SJ, Joo KR, Yang SK, et al. Clinicopathologic features of ileocolonic malignant lymphoma: analysis according to colonoscopic classification. Gastrointest Endosc 2003;57:343-7.

4. Ghai S, Pattison J, Ghai S, et al. Primary gastrointestinal lymphoma: spectrum of imaging findings with pathologic correlation. Radiographics 2007;27:1371-88.

5. Dawson IM, Cornes JS, Morson BC. Primary malignant lymphoid tumours of the intestinal tract: report of 37 cases with a study of factors influencing prognosis. Br J Surg 1961;49:80-9.

6. International Non-Hodgkin's Lymphoma Prognostic Factors Project. A predictive model for aggressive nonHodgkin's lymphoma. N Engl J Med 1993;329:987-94. 